\title{
Acrokeratoelastoidosis as an example of marginal papular acrokeratoderma with prominent elastorrhexis
}

\author{
Magdalena Żychowska , Aleksandra Batycka-Baran², Wojciech Baran² \\ ${ }^{1}$ Department of Dermatology, University of Rzeszow, Rzeszow, Poland \\ 2Department of Dermatology, Venereology and Allergology, Wroclaw Medical University, Wroclaw, Poland \\ Adv Dermatol Venereol 2019; XXXVI (6): 772-774 \\ DOI: https://doi.org/10.5114/ada.2019.91426
}

Acrokeratoelastoidosis (AKE) is a rare condition described by Oswaldo Costa in 1953 [1]. Clinically, it is characterized by the presence of hyperkeratotic or umbilicated papules located on the margins of palms and/or soles. The first lesions usually develop in childhood or early adulthood and gradually increase in size and number, become grouped or form plaques [1-3]. The prominent histological feature of the condition is the diminished density and fragmentation (elastorrhexis) of elastic fibres, which can be detected using Verhoeff-van Gieson or orcein staining [1-3].

A 37-year-old Caucasian woman was admitted to the Department of Dermatology for evaluation of asymptomatic papular lesions located on the margins of hands and feet. The first papules had developed when the patient was 20 years old and had gradually increased in number. The lesions had been initially diagnosed as viral warts and had been treated with keratolytics and several sessions of cryosurgery with no improvement. Family history for similar skin changes was unremarkable. Several years prior to hospitalization, the patient had been diagnosed with ectopic gastric mucosa in the oesophagus, but was otherwise healthy. On physical examination, flesh-coloured papules of 2-4 $\mathrm{mm}$ in diameter were present along the margins of both hands accompanied by slight hyperkeratosis of the palms (Figures

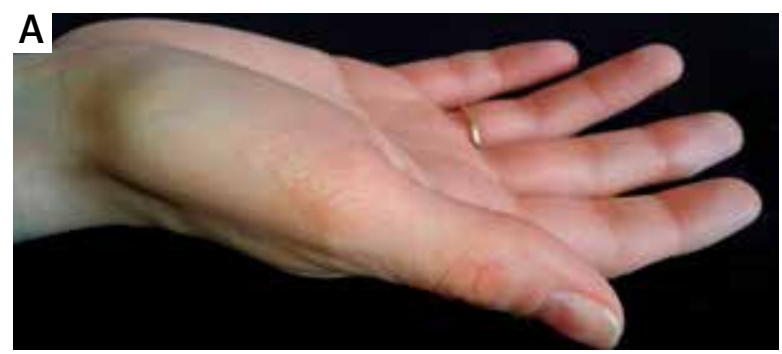

$1 \mathrm{~A}, \mathrm{~B})$. Additionally, several hyperkeratotic papules were present at the line of transgredience between the dorsal and plantar surface of feet. Routine laboratory work-up was within normal limits. A 5-mm punch biopsy was performed from one of the papules, which revealed prominent irregular acanthosis, papillomatosis, hypergranulosis, focal hyperkeratosis and scarce perivascular inflammatory infiltration in the dermis with slight epidermotropism (Figure $2 \mathrm{~A}$ ). Verhoeff-van Gieson staining revealed reduced density of elastic fibres with elastorrhexis (Figure 2 B). On the basis of clinical presentation and histological image the diagnosis of AKE was made. The patient was informed about the benign nature of the condition and she refused to try other treatment options.

AKE is considered to be a very rare disease, but its actual incidence may be underestimated. Over 50 cases have been reported so far. The number of articles has risen significantly in the last decade, with 18 cases reported in 2008-2018. Interestingly, recent reports include predominantly females (a female to male ratio of $7: 2$ ) with late-onset AKE (mainly at 20-30 years of age). The time between the occurrence of first papules and the diagnosis is usually several years, but may be extended up to 20 years. Unilateral lesions have been reported mainly in

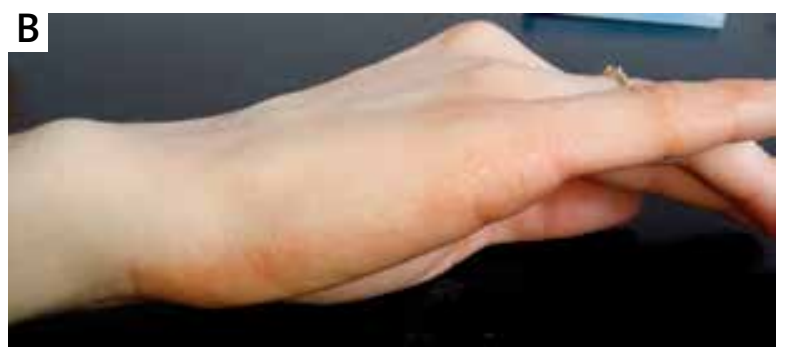

Figure 1. A, B - Shiny flesh-coloured papules located along the line of transgredience between the palmar and dorsal surface of the hand

Address for correspondence: Magdalena Żychowska MD, PhD, Department of Dermatology, University of Rzeszow, 2 Szopena St, 35-055 Rzeszow, Poland, phone: +48 669422 237, email: magda.zychowska@gmail.com Received: 25.04 .2018 , accepted: 1.08 .2018$. 

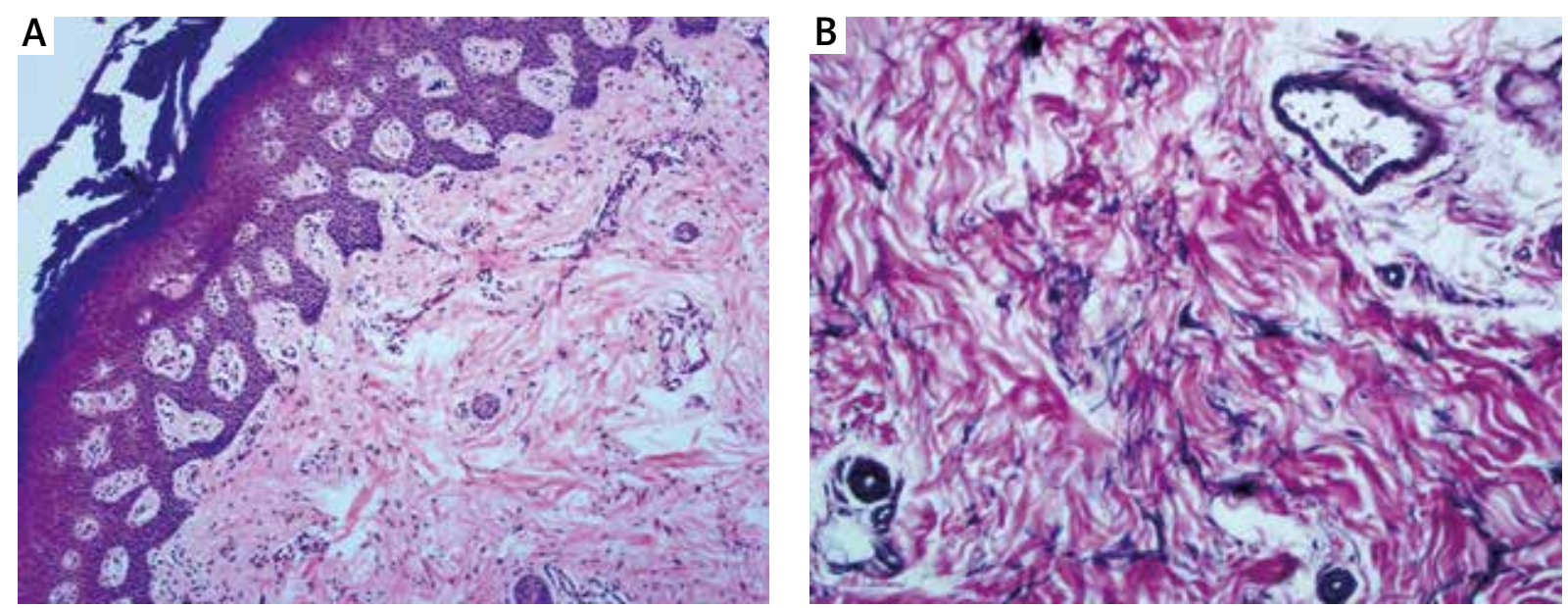

Figure 2. A - Histological findings included irregular acanthosis, papillomatosis, hypergranulosis, focal hyperkeratosis and scarce perivascular inflammatory infiltration in the dermis with slight epidermotropism (40x, hematoxylin and eosin). B - Reduced density of elastic fibres with elastorrhexis (100x, Verhoeff-van Gieson stain)

children and have been present since birth. Both sporadic and familial cases of autosomal dominant inheritance have been reported in the literature [4-9]. A potential linkage to chromosome 2 was suggested by Greiner et al. on the basis of genetic mapping of 21 family members affected by AKE [10]. No particular race predominance has been found so far. The electron microscope (EM) has recently proved to be a valuable tool in making the diagnosis [5]. Fragmented elastic fibres resembling rooster crests can be observed on scanning EM, while transmission EM shows decreased thickness and fragmentation of the elastic fibres, surface indentations, irregular black spots in the fibre core and normal collagen bundles. In single case reports, AKE was associated with nail dystrophy [11] or aquagenic palmoplantar keratoderma [9].

The pathogenesis of the disease remains unknown. One of the most common hypotheses implies a reduced number and impaired function of fibroblasts in the dermis, which secrete abnormal elastic material [12]. An interesting finding was reported by Fiallo et al. [3], who detected fragmentation of the elastic fibres not only in the lesional skin, but also in normal-appearing, non-sunexposed skin, and suggested that AKE may be in fact a primary elastic tissue disease. A confirmation of this hypothesis might be delayed wound healing, which Fiallo et al. observed in one of their patients with AKE [3]. A potential association between late-onset AKE and immunosuppression was suggested by Hussain et al. [6], who reported a patient with AKE developing during therapy with vedolizumab for Crohn's disease.

The nosological position of AKE is not well established, either. The condition was included by Rongioletti et al. [13] in a group of marginal papular acrokeratodermas, which consisted of two acquired entities (degenerative collagenous plaques of the hands and digital papular calcinosis) and five hereditary conditions (AKE, focal acral hyperkeratosis, palmoplantar keratoderma of the punctate type, hereditary papulotranslucent keratoderma and mosaic acral keratosis). All these entities as well as acrokeratosis verruciformis of Hopf and viral warts, should be taken into consideration in differential diagnosis. In case of doubt, histological examination should be performed. The presence of cell vacuolization should raise the suspicion of viral warts, dyskeratosis with "church spire" configuration points to acrokeratosis verruciformis of Hopf, while elastorrhexis is the distinctive histological feature of AKE. Focal acral hyperkeratosis is considered to have the same clinical presentation as AKE, but lacks signs of elastorrhexis on histological examination [14]. Degenerative collagenous plaques of the hands typically develop in middle-aged or older individuals with a history of chronic sun exposure. They are present predominantly on the sides of hands and/or fingers, rarely feet, and are characterized by the presence of degenerative collagen and elastin fibers with extensive actinic damage on histology. Palmoplantar keratoderma of the punctate type presents with dome-shaped papules on the palms and soles, which display hyperkeratosis, parakeratosis, vacuolated epidermis, basal layer spongiosis and dilated sweat ducts on histological examination [2]. On the other hand, Abulafia and Vignale [15] suggested that AKE, focal acral hyperkeratosis and other entities classified by Rongioletti et al. [13] as hereditary marginal papular acrokeratodermas are in fact a result of a variable genetic expression of the same condition and should be considered as clinicopathological variants rather than separate entities.

The prognosis in AKE is very good and for the majority of patients the lesions are only a cosmetic problem. No cases associated with malignancies have been published so far. A few authors reported a potential association of AKE with hyperhidrosis. Nevertheless, in most of the cases, 
the affected patients, as well as the present patient, did not complain of hyperhidrosis of the palms or soles.

The therapy of AKE remains challenging. Cryosurgery or standard keratolytics are usually ineffective. Other attempts to use systemic steroids, methotrexate, dapsone or antibiotics were not successful either [16]. Due to the rarity of the condition, treatment recommendations are mainly based on single cases. Several reports on the efficacy of acitretin or other systemic retinoids in marginal papular acrokeratodermas have been published recently $[4,17,18]$. However, the potential teratogenicity of this therapy should always be taken into consideration in young patients with AKE.

\section{Conflict of interest}

The authors declare no conflict of interest.

\section{References}

1. Costa OG. Acrokeratoelastoidosis. Dermatologica 1953; 107: 164-8.

2. Bogle MA, Hwanq LY, Tschen JA. Acrokeratoelastoidosis. J Am Acad Dermatol 2002; 47: 448-51.

3. Fiallo P, Pesce C, Brusasco A, Nunzi E. Acrokeratoelastoidosis of Costa: a primary disease of the elastic tissue? I Cutan Pathol 1998; 25: 580-2.

4. Lee EA, Kim HS, Kim HO, Park YM. A case of focal acral hyperkeratosis. Ann Dermatol 2009; 21: 426-8.

5. Lopes JF, de Almeida Jr HL, da Cunha Filho RR, et al. Ultrastructure of acrokeratoelastoidosis. J Eur Acad Dermatol 2018; 32: e165-7.

6. Hussain A, Jenkins A, Feneran A, Abdulla F. New-onset acrokeratoelastoidosis in an immunosuppressed patient. JAAD Case Rep 2017; 4: 75-6.

7. Xu CC, Fu LY, Lin D, et al. A case of acrokeratoelastoidosis. Clin Exp Dermatol 2018; 43: 819-20.

8. Uribe P, Ortiz E, Wortsman X, Gonzales S. Acrokeratoelastoidosis of the foot with clinical, dermoscopic, ultrasonographic and histopathologic correlation. J Am Podiatr Med Assoc 2018; 108: 178-81.

9. Poiraud C, Vourch-Jourdain M, Cassagnau E, Barbarot S. Aquagenic palmoplantar keratoderma associated with acrokeratoelastoidosis. Clin Exp Dermatol 2014; 39: 671-2.

10. Greiner J, Krüger J, Palden L, et al. A linkage study of acrokeratoelastoidosis. Possible mapping to chromosome 2. Hum Genet 1983; 63: 222-7.

11. Van Steensel MA, Verstraeten VL, Frank J. Acrokeratoelastoidosis with nail dystrophy: a coincidence or a new entity? Arch Dermatol 2006; 142: 939-41.

12. Masse R, Quillard A, Hery B, et al. Costa acrokerato-elastoidosis. Ultrastructural study. Ann Dermatol Venereol 1977; 104: 441-5.

13. Rongioletti F, Betti R, Crosti C, Rebora A. Marginal papular acrokeratodermas: a unified nosography for focal acral hyperkeratosis, acrokeratoelastoidosis and related disorders. Dermatology 1994; 188: 28-31.

14. Żychowska M, Batycka-Baran A, Woźniak Z, et al. Symmetrical keratotic papules on the hands: a quiz. Acta Derm Venereol 2017; 97: 671-2.

15. Abulafia J, Vignale RA. Degenerative collagenous plaques of the hands and acrokeratoelastoidosis: pathogenesis and relationship with knuckle pads. Int J Dermatol 2000; 39: 424-32.
16. Korc A, Hansen RC, Lynch PJ. Acrokeratoelastoidosis of Costa in North America. A report of two case. J Am Acad Dermatol 1985; 12: 832-6.

17. Lambiris AG, Newman PL Marginal papular acrokeratodermas: no racial limitations for a clinical spectrum that responds to acitretin. Dermatology 2001; 203: 63-5.

18. Handfield-Jones S, Kennedy CT. Acrokeratoelastoidosis treated with etretinate. J Am Acad Dermatol 1987; 17: 881-2. 